\title{
Estudo da influência do ar no movimento de queda dos corpos: uma comparação entre a previsão teórica e os dados experimentais usando o Tracker
}

Study of the influence of air on the falling motion of bodies: a comparison between theoretical prediction and experimental data using the Tracker

\author{
Marcos Roberto Rossini*1, Marcio José Alves ${ }^{1}$, Luís Henrique Amorin ${ }^{1,2}$, \\ Paulo Sérgio de Camargo Filho ${ }^{1}$
}

\footnotetext{
${ }^{1}$ Universidade Tecnológica Federal do Paraná, Departamento Acadêmico de Física - Campus Londrina, Londrina, PR, Brasil

${ }^{2}$ Universidade Federal da Bahia, Instituto de Ciência, Tecnologia e Inovação, Camaçari, BA, Brasil
}

\author{
Recebido em 15 de julho de 2020. Revisado em 20 de agosto de 2020. Aceito em 01 de setembro de 2020.
}

\begin{abstract}
Esse artigo descreve uma proposta de atividade prática para a graduação, envolvendo um estudo teóricoexperimental sobre a influência do ar no movimento de queda dos corpos. Para isso, analisou-se a dinâmica do movimento de objetos esféricos em queda vertical no ar, considerando-se a força de arrasto, apreciando-se a relação entre um intervalo de tempo característico e o comportamento da velocidade e da aceleração, comparandose a previsão do modelo teórico com os dados experimentais. Para a obtenção dos dados, realizou-se um ensaio utilizando-se de filmagem da queda com aparelho celular e posterior tratamento com o Tracker. Os resultados apontaram que o movimento de queda de um corpo esférico de isopor com diâmetro da ordem de dois centímetros serviria ao propósito, permitindo verificar-se a presença do arrasto inercial e a tendência a uma velocidade limite em uma queda inferior a três metros, em acordo com as previsões teóricas. Além disso, verificou-se não haver correlação entre o módulo da velocidade inicial com a magnitude da velocidade terminal, implicando que a transição ocorrida do regime viscoso para o inercial, nos primeiros instantes, não afetaria o comportamento da velocidade nos instantes seguintes.
\end{abstract}

Palavras-chave: Ensino de Física, queda no ar, velocidade terminal, força de arrasto, resistência do ar, viscosidade.

This article describes a practical activity proposal for graduation, involving a theoretical and experimental study on the influence of air on the falling motion of bodies. For this, the dynamics of the movement of spherical objects in vertical fall in the air were analyzed, considering the drag force, appreciating the relationship between a characteristic time interval and the behavior of speed and acceleration, comparing the prediction of the theoretical model with the experimental data. To obtain the data, a test was performed using video footage of the fall with a cell phone and subsequent treatment with the Tracker. The results showed that the falling movement of a spherical styrofoam body with a diameter of the order of two centimeters would serve the purpose, allowing to verify the presence of inertial drag and the tendency to a limit speed in a fall of less than three meters, in according to theoretical predictions. In addition, it was found that there is no correlation between the initial velocity module and the magnitude of the terminal velocity, implying that the transition from the viscous to the inertial regime, in the first moments, would not affect the behavior of the velocity in the following moments.

Keywords: Physics teaching, air fall, terminal speed, drag force, air resistance, viscosity.

\section{Introdução}

A consideração do efeito do ar em movimentos de queda e lançamentos é extremamente relevante, e certamente indispensável na dinâmica da sustentação do voo [1]. Precipitação de granizo [2], transporte de grãos empregando fluxo de ar [3], lançamento de bolas, salto de atletas, movimento de foguetes e de projéteis são fenômenos cotidianos cuja descrição mecânica somente apresentará resultados satisfatórios quando consideradas as forças estáticas e dinâmicas presentes no movimento

\footnotetext{
*Endereço de correspondência: rossini@utfpr.edu.br
}

de corpos em meios viscosos 4-11. Nos livros mais tradicionais utilizados nos cursos de Física [12 [15], rara e timidamente aborda-se a força de arrasto. Nestes, as transposições didáticas não levam em consideração formas mais adequadas para o tratamento, em nível introdutório, do estudo do movimento em meio viscoso para estudantes dos cursos superiores em ciências exatas, especialmente no Curso de Física. Há de se considerar ainda que, em tais abordagens, encontra-se a força de arrasto nos lançamentos descrita como diretamente proporcional ao módulo da velocidade [16 18. Como se pretende explorar nas próximas seções, o movimento de queda de um corpo esférico de isopor, com diâmetro 
da ordem de dois centímetros, pode ser melhor descrito quando a força de arrasto é considerada proporcional ao quadrado do módulo da velocidade.

$\mathrm{Na}$ esteira dessas discussões, o estudo visa realizar um aprofundamento teórico a respeito do movimento em meio viscoso de corpos em queda no ar, apreciando a relação entre um intervalo de tempo característico e o comportamento da velocidade e da aceleração, comparando o modelo teórico com os dados experimentais obtidos com equipamentos de baixo custo. Para isso, consideram-se técnicas e equipamentos acessíveis que permitem a reprodutibilidade em qualquer laboratório didático de Física. Assim, optou-se pelo emprego de telefones celulares para a coleta de dados por meio de filmagens das quedas, os quais foram inicialmente tratados com o emprego do programa Tracker ${ }^{\odot}$ [19], e posteriormente com softwares de análise de dados [18, 19 .

\section{Abordagem teórica da queda vertical no ar com força de arrasto inercial}

Em trajetórias relativamente curtas, próximas à superfície da Terra, é possível considerá-la referencial inercial, desprezando-se a força de Coriolis [12]. A dinâmica na presença do campo gravitacional terrestre envolve, naturalmente, a força peso. Havendo movimento relativo entre o corpo e o fluido no qual está imerso, sua superfície submete-se a ação de forças normais, responsáveis pela pressão, e de forças tangenciais, associadas à viscosidade do fluido e responsáveis por tensões de cisalhamento [20]. Tomando o ar como fluido que preenche o meio, desconsidera-se o empuxo causado pelo gradiente de pressão hidrostática [21], visto ser muito menor que o peso do corpo. Para um corpo totalmente imerso, a razão entre os módulos do seu peso e do empuxo, $P / E$, é dada por

$$
\frac{P}{E}=\frac{\rho_{c} \cdot V_{c} \cdot g}{\rho_{f} \cdot V_{c} \cdot g} \rightarrow \frac{P}{E}=\frac{\rho_{c}}{\rho_{f}}
$$

sendo $V_{c}$ o volume corpo, $\rho_{c}$ sua densidade, $\rho_{f}$ a densidade do fluido e $g$ o módulo da aceleração gravitacional. Para o ar, a $20,0^{\circ} \mathrm{C}$ e $566 \mathrm{~m}$ de altitude, considerou-se $\rho_{f}=1,17 \mathrm{~kg} / \mathrm{m}^{3}$. Nos ensaios, o material menos denso que se utilizou foi o isopor, $\rho_{\text {iso }}=41,2 \mathrm{~kg} / \mathrm{m}^{3}$, e o mais denso foi o plástico, $\rho_{\text {plást }}=894,0 \mathrm{~g} / \mathrm{cm}^{3}$, significando que o menor valor da razão seria $P / E=35,2$. A título de comparação, caso se considere o granizo, $\rho=910 \mathrm{~kg} / \mathrm{m}^{3}$, a razão será $P / E=778$.

Cuidou-se para que a prática ocorresse em ambiente livre da ação de ventos. Assim, a velocidade relativa entre o fluido e o corpo abandonado é simplesmente sua velocidade, essencialmente vertical em relação à superfície da Terra, porque se pressupõe que o ar esteja em repouso neste referencial. Não havendo rotação do objeto em queda, pode desprezar-se o efeito Magnus [21. Com estas simplificações, analisou-se apenas a componente resultante das forças hidrodinâmicas que resistem ao movimento de translação na direção e sentido oposto ao escoamento, denominada força de arrasto, $\vec{F}_{\text {arr }}$. Ela se deve ao arrasto inercial, $\vec{F}_{\text {in }}$, gerado pelas forças normais, e ao arrasto viscoso, $\vec{F}_{\text {vis }}$, mas, nos ensaios deste trabalho, preponderaram as forças de arrasto inerciais. O que permite tal afirmativa é o cálculo da razão $R e=$ $F_{i n} / F_{v i s}$, conhecida como número de Reynolds [22]:

$$
R e=\frac{\rho_{f} \cdot v \cdot L}{\eta}
$$

onde $v$ é a velocidade do corpo em relação ao fluido, $L$ é uma dimensão característica, no caso da esfera esta dimensão é seu diâmetro $D$, e $\eta$ a viscosidade dinâmica do fluido. Para o ar a $20,0^{\circ} \mathrm{C}$ e 1,00 atm, adotou-se $\eta=2,00.10^{-5}$ Pa.s [3], embora este valor, assim como o valor da densidade do ar, são parâmetros que dependem de outras variáveis, como pressão atmosférica e temperatura, para ajustes finos [23], mas não são necessários no escopo deste trabalho.

Conforme um objeto cai, o módulo de sua velocidade aumenta pela ação gravitacional, enquanto o módulo de sua aceleração diminui gradativamente porque o módulo da força de arrasto aumenta com o incremento da velocidade. Quando a força de arrasto tiver a mesma magnitude da força peso, a velocidade atingirá seu valor máximo, conhecido como velocidade terminal, $v_{t}$. No Quadro 1, apresentam-se algumas estimativas do número de Reynolds para alguns objetos em queda no ar, supondo-se algumas velocidades terminais e diâmetros.

A intensidade da força de arrasto [2, 3, 5, 8, é dada pela expressão:

$$
F_{\text {arr }}=\frac{C \cdot \rho_{f} \cdot A \cdot v^{2}}{2}
$$

onde $C$ é o coeficiente de arrasto, $\rho_{f}$ é a densidade do fluido, $A$ é a área da seção transversal do corpo na direção normal à velocidade relativa, $\vec{v}$. O valor do coeficiente de arrasto para esferas lisas já foi bastante estudado [2, 5, 24]. Para $10^{3}<R e<10^{5}$, que é um intervalo coerente com a simulação apresentada no Quadro 1] temse um coeficiente praticamente constante, e encontramse na literatura valores tais que o coeficiente situa-se no intervalo $(0,42 ; 0,50)$ [5, 25]. Assim, para a força de arrasto inercial toma-se:

$$
F_{i n}=\frac{C \cdot \rho_{f} \cdot A \cdot v^{2}}{2}
$$

Quadro 1: Número de Reynolds, Re, para esferas em queda no ar, considerando hipoteticamente alguns diâmetros, D, e valores de velocidades terminais, $v_{t}$ [2].

\begin{tabular}{|l|c|c|}
\hline $\mathrm{D}(\mathrm{cm})$ & $v_{t}(\mathrm{~m} / \mathrm{s})$ & $\mathrm{Re}$ \\
\hline 1,0 & 1,0 & $6.10^{2}$ \\
\hline 1,0 & 10,0 & $6.10^{3}$ \\
\hline 5,0 & 10,0 & $3.10^{4}$ \\
\hline 5,0 & 30,0 & $9.10^{4}$ \\
\hline
\end{tabular}


assumindo-se que o coeficiente de arrasto seja constante. É justamente esta dependência da força de arrasto com o quadrado do módulo da velocidade, que raramente se encontra nos livros didáticos.

A força de arrasto viscoso é significante para situações onde o número de Reynolds, equação (2), é relativamente pequeno $(R e<1)$. Isto procede quando partículas deslocam-se com baixas velocidades através de fluidos viscosos, como micro-organismos movendo-se na água, grãos de poeira no ar, e gotículas de óleo em suspensão no ar, como no experimento de Millikan [25, 26]. Nestas condições, $R e<1$, Figura 1. o coeficiente de arrasto de corpos esféricos pode ser dado em função do número de Reynolds, com muito boa aproximação, através de uma expressão muito simples:

$$
C=\frac{24}{R e}=\frac{24}{\frac{\rho_{f} \cdot v \cdot 2 R}{\eta}} \rightarrow \quad C=\frac{12 \eta}{\rho_{f} \cdot v \cdot R}
$$

mostrando que o aumento da velocidade reduzirá o coeficiente de arrasto. Substituindo-se esta nova expressão do coeficiente na equação (3), tem-se a expressão do arrasto viscoso de Stokes para corpos esféricos:

$$
F_{v i s}=\frac{12 \eta}{\rho_{f} \cdot v \cdot R} \cdot \frac{\rho_{f} \cdot\left(\pi R^{2}\right) \cdot v^{2}}{2} \rightarrow \quad F_{v i s}=6 \pi \eta R \cdot v
$$

Com o entendimento das forças mais significativas para se descrever o movimento dos corpos em queda no ar, encontra-se a intensidade da força resultante sobre os corpos esféricos empregados nos ensaios. Sendo o movimento unidimensional, dispensou-se a notação vetorial, e simplesmente escreveu-se para a força resultante:

$$
F_{R}=P-F_{i n}-F_{v i s}-E
$$

mas, conforme análise anterior, o empuxo e o arrasto viscoso poderão ser desconsiderados. Via de regra, não havendo sustentação, o efeito do arrasto viscoso é desprezível, e são poucas as situações onde o arrasto inercial e o arrasto viscoso atuarão com a mesma ordem de grandeza [20, 27]. Assim, a segunda Lei de Newton

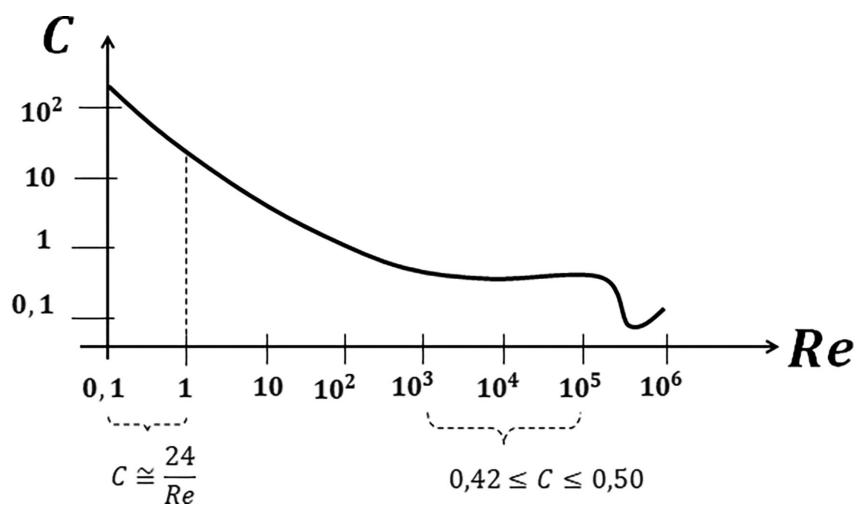

Figura 1: Esboço do gráfico do coeficiente de arrasto para esferas lisas em função do número de Reynolds [25]. permite obter a aceleração do corpo:

$$
m . a=P-F_{\text {in }}
$$

Para corpos esféricos, secção transversal $A=\pi \cdot D^{2} / 4$, as relações (4) e (8) permitem que se obtenha uma nova equação relacionando a aceleração e a velocidade:

$$
m . a=m . g-\frac{C \cdot \rho_{f} \cdot \frac{\pi \cdot D^{2}}{4} \cdot v^{2}}{2} \rightarrow a=g-k \cdot v^{2}
$$

onde

$$
k=\frac{C \cdot \rho_{f} \cdot \pi \cdot D^{2}}{8 \cdot m}
$$

e substituindo-se a massa do corpo esférico por $\frac{4}{3} \pi \cdot\left(\frac{D}{2}\right)^{3} \cdot \rho_{c}$ :

$$
k=\frac{3}{4} \cdot C \cdot \frac{\rho_{f}}{\rho_{c}} \cdot \frac{1}{D}
$$

A equação (9) mostra que o módulo da aceleração é máximo quando $v=0$, e diminui à medida que o valor da velocidade aumenta, conforme a discussão preliminar. Para um objeto abandonado, ou lançado para baixo com velocidade inferior à velocidade terminal, a força de arrasto inercial será menor que seu peso e, consequentemente, o módulo da velocidade do corpo aumentará. Quando o peso e a força de arrasto se equilibram, a velocidade terminal é atingida, resultando um movimento retilíneo e uniforme. Tomando-se a equação (9), com $v=v_{t}$ e $a=0$, obtém-se a expressão para a velocidade terminal

$$
v_{t}=\sqrt{\frac{g}{k}}
$$

ou, com a equação 11,

$$
v_{t}=\sqrt{\frac{4}{3 C} \cdot \frac{\rho_{c}}{\rho_{f}} \cdot D \cdot g}
$$

que traz algumas informações bem interessantes. A aceleração gravitacional e a densidade do corpo contribuem favoravelmente para o incremento da velocidade terminal, visto que aumentam a força peso, enquanto a densidade do fluido e o coeficiente de arrasto dificultam sua mobilidade. Pode parecer estranho o diâmetro do corpo favorecer a velocidade terminal, porque um diâmetro maior implica que há uma secção transversal maior e, consequentemente, aumento do arrasto. Esta impressão se desfaz quando se lembra de que a massa e o peso do corpo são proporcionais ao cubo do diâmetro, enquanto o a força de arrasto é proporcional ao quadrado.

Empregando-se a equação 12 é possível escrever-se a equação 9 de um modo mais elegante:

$$
a=g \cdot\left[1-\left(\frac{v}{v_{t}}\right)^{2}\right]
$$



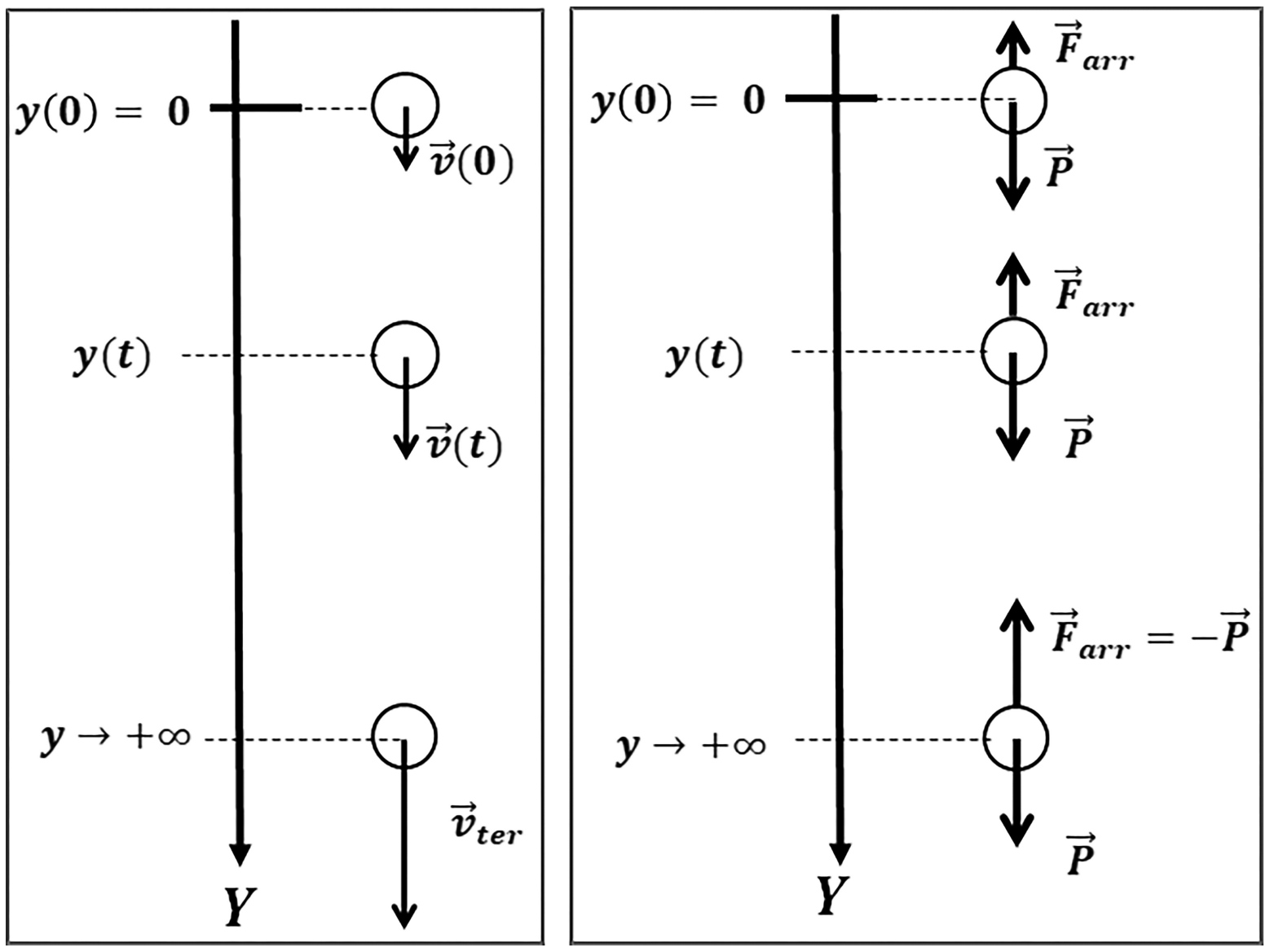

Figura 2: Referencial adotado para o estudo do movimento vertical de corpos esféricos em queda no ar. À esquerda, diagrama representando a velocidade e a posição em função do tempo. À direita, o diagrama das forças correspondentes.

ou, em termos de uma equação diferencial,

$$
\frac{d v}{d t}=g \cdot\left[1-\left(\frac{v}{v_{t}}\right)^{2}\right]
$$

resolvida separando-se as variáveis e adotando-se o referencial representado na Figura 2.

$$
\begin{aligned}
\frac{d v}{1-\left(\frac{v}{v_{t}}\right)^{2}} & =g \cdot d t \rightarrow \int_{v_{0}}^{v(t)} \frac{d v}{1-\left(\frac{v}{v_{t}}\right)^{2}} \\
& =\int_{0}^{t} g \cdot d t^{\prime} \rightarrow \cdots \rightarrow \\
v(t) & =v_{t} \cdot \tanh \left(\frac{t}{\tau}+\frac{\theta}{2}\right)
\end{aligned}
$$

sendo $\tau$ o intervalo de tempo característico do movimento, citado anteriormente, agora definido como

$$
\tau=\frac{v_{t}}{g}
$$

e

$$
\theta=\ln \left(\frac{v_{t}+v_{0}}{v_{t}-v_{0}}\right)
$$

cuidando para que $v_{t}-v_{0}>0$. Com as equações (14) e (16), encontra-se uma expressão para a aceleração em função do tempo:

$$
a=g \cdot\left\{1-\left[\tanh \left(\frac{t}{\tau}+\frac{\theta}{2}\right)\right]^{2}\right\}
$$

Partindo-se da equação (16), também se obteve a equação da posição em função do tempo:

$$
\begin{aligned}
\frac{d y}{d t} & =v_{t} \cdot \tanh \left(\frac{t}{\tau}+\frac{\theta}{2}\right) \rightarrow \int_{0}^{y(t)} d y \\
& =v_{t} \cdot \int_{o}^{t} \tanh \left(\frac{t^{\prime}}{\tau}+\frac{\theta}{2}\right) \cdot d t^{\prime} \rightarrow \cdots \rightarrow \\
y(t) & =\frac{v_{t}^{2}}{g} \cdot \ln \left[\frac{\cosh \left(\frac{t}{\tau}+\frac{\theta}{2}\right)}{\cosh \left(\frac{\theta}{2}\right)}\right]
\end{aligned}
$$

Para melhor entendimento do significado do intervalo de tempo característico, $\tau=v_{t} / g$, considerou-se nula a velocidade inicial. Tomando-se a equação 18 com 

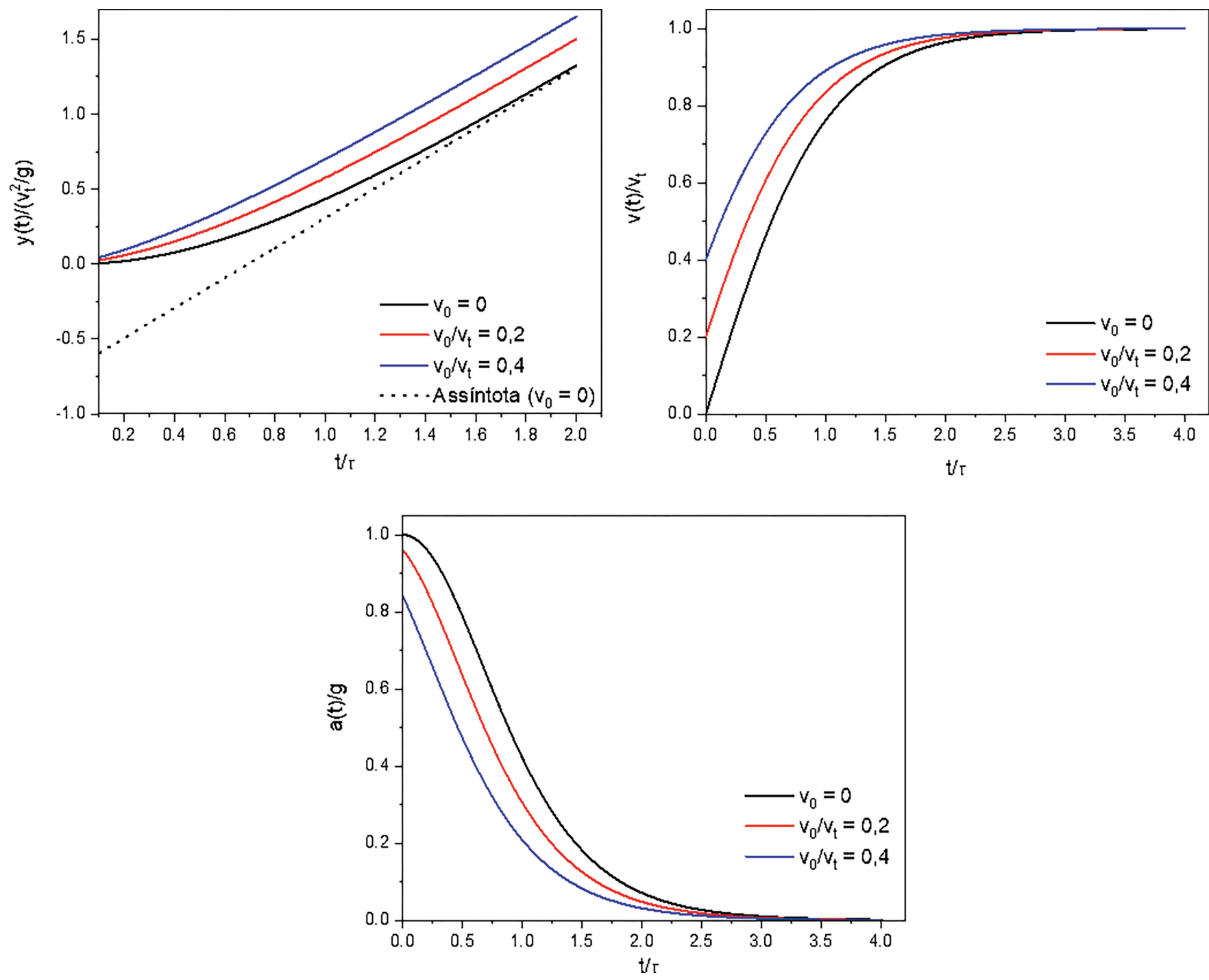

Figura 3: Gráficos das equações cinemáticas da posição, equações (20), (21) e (24), da velocidade, equações (16) e (22), e da aceleração, equações (19) e 23), em função de $\tau$. Pode-se observar no gráfico da posição as curvas tendendo para a reta de um Movimento Uniforme (assíntota), o que equivale a dizer que $\mathrm{v} \rightarrow \mathrm{v}$-t e a $\rightarrow 0$ para $\mathrm{t} \rightarrow \infty$.

$v_{0}=0 \rightarrow \theta=0$, reescreveram-se as equações 20, , 16 . e (19), respectivamente:

$$
\begin{aligned}
y(t) & =\frac{v_{t}^{2}}{g} \cdot \ln \left[\cosh \left(\frac{t}{\tau}\right)\right] \\
v(t) & =v_{t} \cdot \tanh \left(\frac{t}{\tau}\right) \\
a & =g \cdot\left\{1-\left[\tanh \left(\frac{t}{\tau}\right)\right]^{2}\right\}
\end{aligned}
$$

e simulou-se o comportamento da velocidade e da aceleração em função do intervalo de tempo característico, conforme apresentado na Figura 3 e no Quadro 2. Também é possível observar-se que, para grandes valores do tempo, a assíntota da equação da posição é uma equação de movimento uniforme:

$$
\begin{aligned}
y(t)= & \frac{v_{t}^{2}}{g} \cdot\left\{\ln \left[\frac{e^{-\left(\frac{t}{\tau}+\frac{\theta}{2}\right)}+e^{+\left(\frac{t}{\tau}+\frac{\theta}{2}\right)}}{2}\right]\right. \\
& \left.-\ln \left[\cosh \left(\frac{\theta}{2}\right)\right]\right\}
\end{aligned}
$$

de modo que, para $t \gg \tau$, pode-se fazer a aproximação

$$
\begin{aligned}
y(t) \cong & \frac{v_{t}^{2}}{g} \cdot\left\{\ln \left[\frac{e^{+\left(\frac{t}{\tau}+\frac{\theta}{2}\right)}}{2}\right]\right. \\
& \left.-\ln \left[\cosh \left(\frac{\theta}{2}\right)\right]\right\} \rightarrow \cdots \rightarrow \\
y(t) \cong & -\frac{v_{t}^{2}}{g} \cdot \ln \left[2 \cdot \cosh \left(\frac{\theta}{2}\right)\right]+v_{t} \cdot t
\end{aligned}
$$


Quadro 2: Valores da velocidade e da aceleração de um corpo esférico em função do intervalo de tempo característico, $\tau=$ $v_{t} / g$, considerando-se velocidade inicial nula e queda vertical na presença do ar com força de arrasto inercial.

\begin{tabular}{|l|l|l|}
\hline $\mathrm{t} / \tau$ & $v / v_{t}$ & $a / g$ \\
\hline 0 & 0,00 & 1,00 \\
\hline 1 & 0,76 & 0,42 \\
\hline 2 & 0,96 & 0,07 \\
\hline 3 & 0,99 & 0,01 \\
\hline
\end{tabular}

Observe-se no Quadro 2 que, para três tempos característicos, o objeto em queda já se encontra a $99 \%$ da velocidade terminal, com aceleração da ordem de $0,1 \mathrm{~m} / \mathrm{s}^{2}$, e seu movimento aproxima-se do Movimento Uniforme, como pode ser visualizado nos gráficos da Figura 3 Estas previsões teóricas explicaram os resultados experimentais para uma esfera de isopor, conforme os dados que serão apresentados na próxima seção.

\section{Aquisição de Dados Experimentais para Estudo do Efeito da Força de Arrasto Sobre o Movimento de Queda "livre", Usando o Tracker}

Propõe-se um experimento de queda livre que considera o efeito de arrasto do ar sobre corpos esférico com emprego de vídeo-análise [28. 29]. Nesta atividade, utiliza-se materiais de baixo custo facilmente disponíveis e exploram-se recursos tecnológicos acessíveis. Essencialmente, o experimento consistiu em filmar-se a queda vertical de dois corpos esféricos com diâmetros da ordem de alguns centímetros, abandonando ambos da mesma altura, registrando o movimento de queda vertical sobre influência da aceleração da gravidade e da força de arrasto do ar. Para o tratamento das imagens, empregou-se o Tracker $^{\odot}$, um software livre de modelagem computacional desenvolvido por Douglas Brown, colaborador do projeto Open Source Physics (OSP). Através da vídeo-análise de objetos que podem ser considerados pontos materiais, este programa permite a obtenção de dados cinemáticos de fenômenos físicos filmados 30 33.

Nos ensaios foram utilizados dois corpos esféricos, um constituído por plástico, diâmetro $(3,53 \pm 0,04) \mathrm{cm}$ e massa $(20,590 \pm 0,001) \quad g$, o outro por de isopor, diâmetro $(2,35 \pm 0,10) \mathrm{cm}$ e massa $(0,280 \pm 0,001) \mathrm{g}$. A filmagem desempenhou processo central para análise. Antes, definiu-se o celular e o ambiente onde foram gravadas as quedas. Iniciaram-se as filmagens com um aparelho que gravava a 30 quadros por segundo (fps), bastante comum no mercado, e suficiente para a análise do movimento da esfera de isopor. Quando se realizaram os ensaios para a esfera de plástico, cuja velocidade terminal é maior que a da esfera de isopor, substituiu-se o celular por outro que grava a uma taxa de $120 \mathrm{fps}$.

No processo de calibração empregou-se uma barra de referência vertical, conforme a Figura 4 Nestas tomadas de dados, a aresta da coluna foi usada como linha vertical. Este procedimento permitiu que plano definido pelos eixos de coordenadas coincidisse com a face da coluna, onde se fixou a barra de referência para
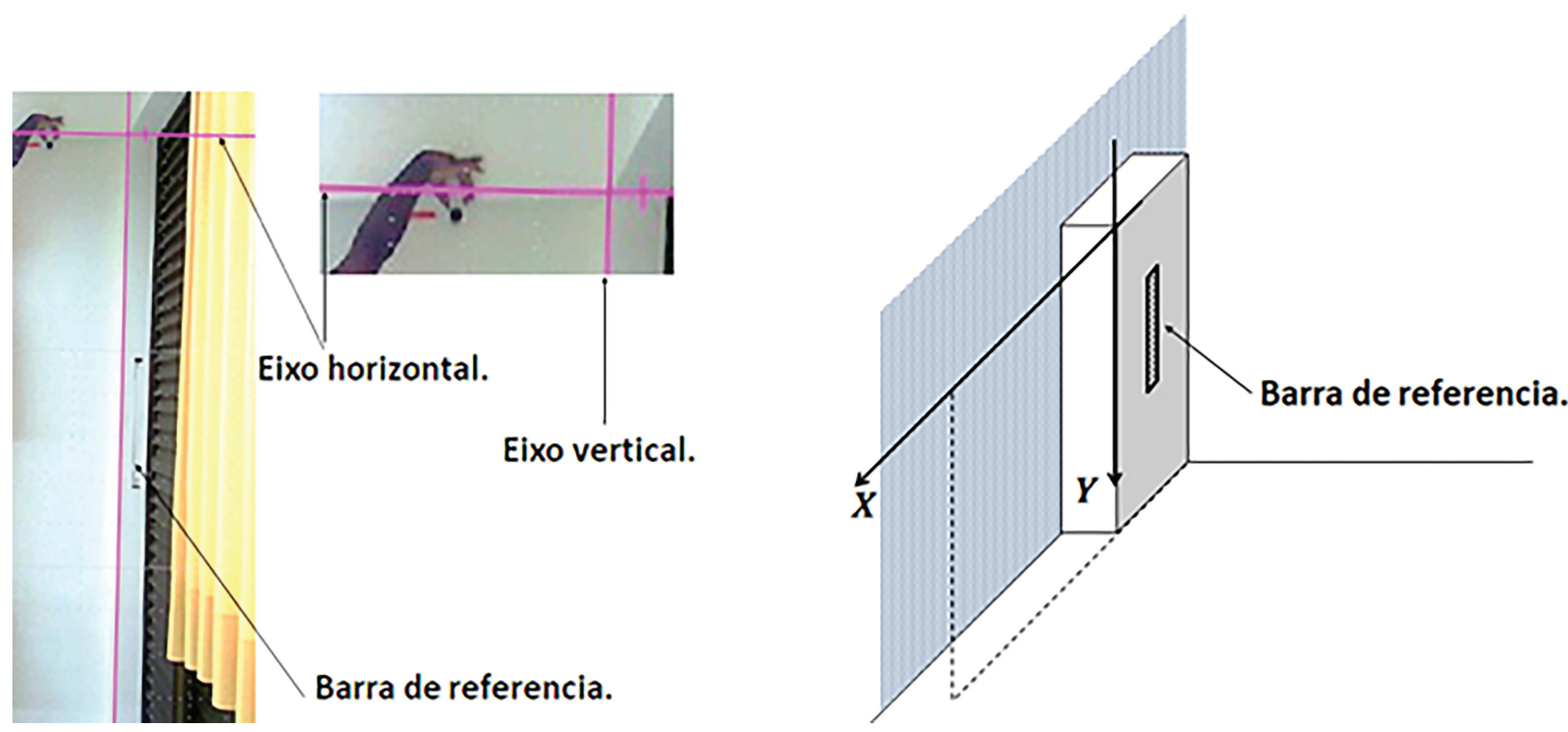

Figura 4: À esquerda, recorte do início de uma cena gravada, antes de a bola ser abandonada, com detalhe para a mão do experimentador e os eixos horizontais e verticais que contêm a trajetória. À direita, perspectiva da sala, mostrando a coluna em ressalto na parede. 
calibração das distâncias, minimizando-se os problemas de paralaxe [34, 35].

O ponto material escolhido para análise foi o centro de massa dos corpos esféricos. No Tracker ${ }^{\odot}$, a determinação destes pontos ao longo da trajetória foi feita de forma manual. Assim minimizaram-se os erros grosseiros e sistemáticos [34, 36], optando-se por não se utilizar da aquisição automática de dados, visto que, nos quadros filmados, os corpos deixam rastros que aumentam de comprimento durante a queda. Isso se deve ao intervalo de tempo constante que celular leva para filmar cada quadro e ao aumento da velocidade do corpo. Os dados experimentais $\left(y_{i}, t_{i}\right)$ foram organizados em uma planilha do $C a l c^{\odot}$, onde também se geraram os valores $\left(y\left(t_{i}\right), t_{i}\right)$, previstos pelos dos modelos teóricos.

\section{Análise de dados}

Conforme descrito na seção anterior, foram realizados testes com dois corpos esféricos distintos, efetuando-se duas dezenas de lançamentos para cada, considerandose inicialmente que a velocidade inicial dos corpos era nula. Os ensaios começaram com a esfera de plástico, e construíram-se gráficos da posição em função do tempo para se confrontar os dados experimentais com modelo teórico, equação 21 e Figura 5 Nos gráficos que seguem, as curvas do modelo teórico foram geradas utilizando-se parâmetros previamente escolhidos e superpostos aos dados experimentais da vídeo-análise. Assim, enfatiza-se que se adotou nas simulações $\rho_{f}=$ $1,17 \mathrm{~kg} / \mathrm{m}^{3}, \eta=2,00 \cdot 10^{-5}$ Pa.s, $C=0,45$ e $g=$ $9,786 \mathrm{~m} / \mathrm{s}^{2}$. O valor da aceleração gravitacional foi calculado com base na equação

$$
\begin{aligned}
g(L, H)= & 9,80613 \cdot\left(1-2,6325 \cdot 10^{-3} \cdot \cos 2 L\right) \\
& \left(1-\frac{3,92 \cdot 10^{-7}}{m} \cdot H\right) \frac{m / s}{s}
\end{aligned}
$$

sendo $L$ a latitude dada em graus, e $\mathrm{H}$ a altitude em metros [37. Para a cidade de Londrina-PR, considerouse $L=-23,33^{\circ}$ e $H=566 m$ 38. Para se avaliar o desvio relativo médio entre o modelo teórico e os dados experimentais, calculou-se o desvio relativo médio

$$
\left\langle D R_{y}\right\rangle=\frac{1}{N} \cdot \sum_{i=1}^{N}\left|\frac{y_{i}-y\left(t_{i}\right)}{y\left(t_{i}\right)}\right|
$$

desconsiderando-se o instante $t_{0}=0$ porque $y_{0}=y(0)=$ 0 , encontrando-se $\left\langle D R_{y}\right\rangle=59 \%$.

Suspeitou-se que a discordância entre os dados experimentais e as curvas teóricas do gráfico (Figura 5 p ocorria porque a velocidade inicial poderia ser diferente de zero. Assim, houve necessidade de testarem-se os modelos dados pelas equações 16 e 22 . O cálculo numérico da velocidade no instante $t_{n}>0, n=1 \ldots N$, pôde ser feito empregando-se os dados experimentais da posição.

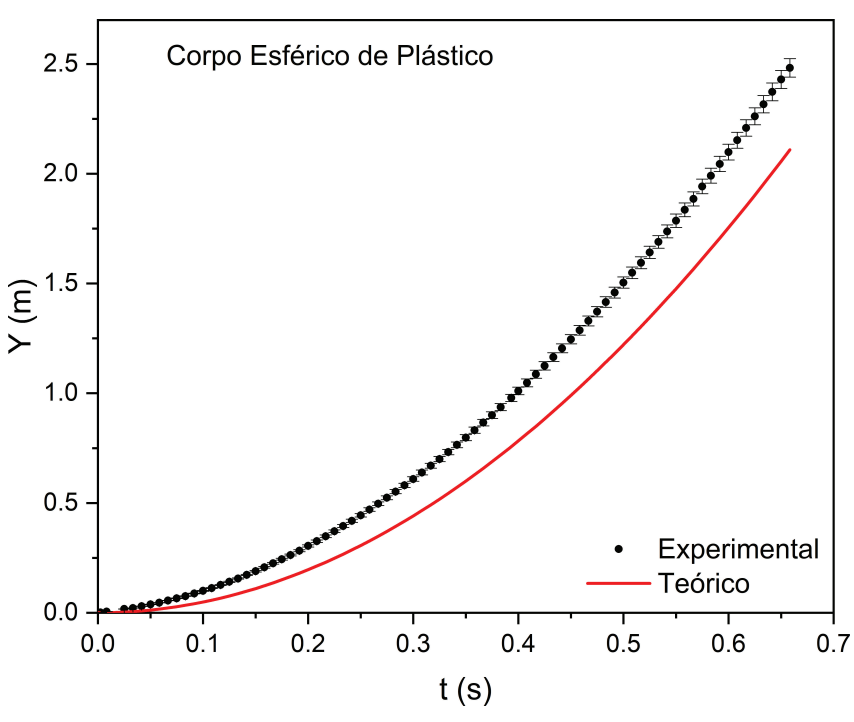

Figura 5: Gráfico da posição versus tempo para um lançamento do corpo esférico de plástico, confrontando previsão teórica, linha inferior, com os dados experimentais. Gravação a 120 fps.

Admitindo-se que a aceleração não variasse significativamente no pequeno intervalo de tempo $\left[t_{n+1} ; t_{n-1}\right]$, entre três tomadas de dados consecutivas, tratou-se o movimento neste intervalo como uniformemente variado, e a velocidade média seria igual à média das velocidades, sendo verdadeira a igualdade:

$$
\begin{aligned}
\frac{y_{n}-y_{n-1}}{t_{n}-t_{n-1}}+\frac{y_{n+1}-y_{n}}{t_{n+1}-t_{n}}= & \frac{v\left(t_{n-1}\right)+v\left(t_{n}\right)}{2} \\
& +\frac{v\left(t_{n}\right)+v\left(t_{n+1}\right)}{2}
\end{aligned}
$$

onde

$$
\begin{aligned}
& v\left(t_{n-1}\right)=v\left(t_{n}\right)-a \cdot\left(t_{n}-t_{n-1}\right) \quad \mathrm{e} \\
& v\left(t_{n+1}\right)=v\left(t_{n}\right)+a \cdot\left(t_{n+1}-t_{n}\right)
\end{aligned}
$$

e como os intervalos de tempo são todos iguais, $t_{n}-$ $t_{n-1}=t_{n+1}-t_{n}$, obtém-se um resultado simples:

$$
v\left(t_{n}\right)=\frac{y_{n+1}-y_{n-1}}{t_{n+1}-t_{n-1}}
$$

equivalente a dizer que a velocidade no instante $t_{n}$ é igual a velocidade média no intervalo $\left[t_{n+1} ; t_{n-1}\right]$. Aplicando-se este algoritmo aos dados experimentais, obtiveram-se as velocidades, apresentando-se um resultado na Figura 6 .

O gráfico da Figura 6 corroborou com a existência de uma velocidade inicial não nula. O ajuste dos dados experimentais com um polinômio de primeira ordem no tempo mostrou-se satisfatório para fornecer a velocidade inicial. Os gráficos gerados considerando-se velocidade inicial não nula estão representados nas Figuras 7, 8, 9 e 10 onde se apresenta o resultado para um lançamento da esfera de plástico, $v_{0}=0,55 \mathrm{~m} / \mathrm{s}$, e outro para a esfera 


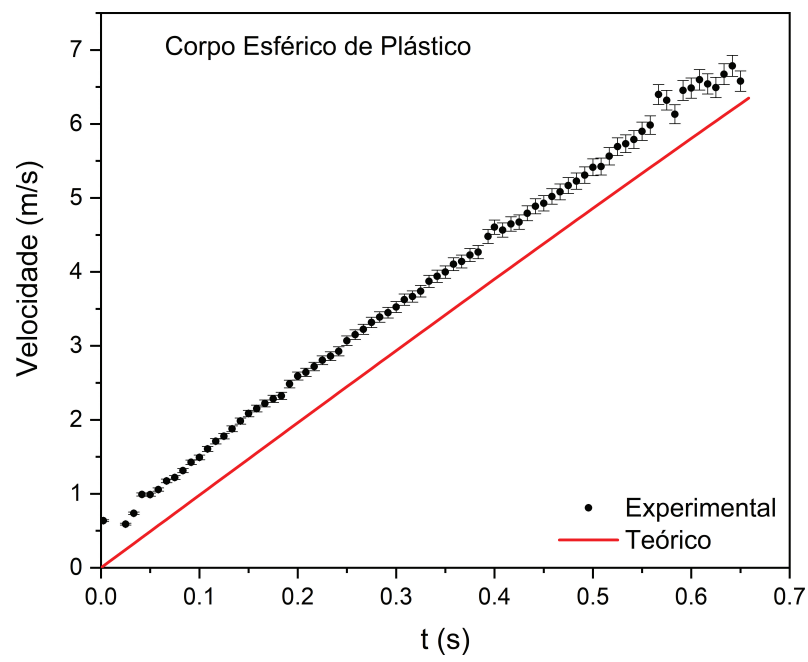

Figura 6: Gráfico comparativo da velocidade em função do tempo, considerando velocidade inicial nula. A curva superior corresponde aos valores da velocidade obtidos aplicando-se aos dados experimentais a equação (27). Gravação a $120 \mathrm{fps}$.
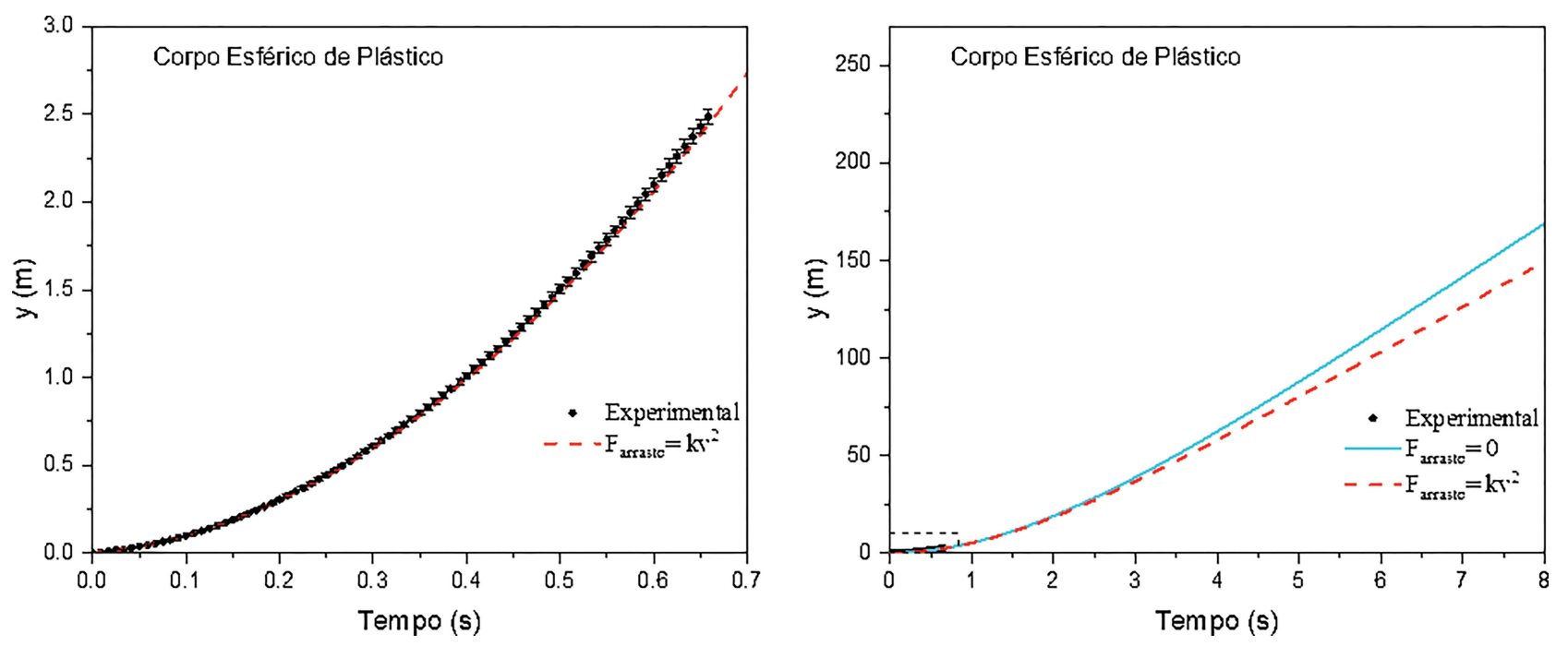

Figura 7: Gráficos comparativos da posição em função do tempo para a o corpo esférico de plástico. À direita, extrapolação das curvas teóricas.

de isopor, $v_{0}=-0,40 \mathrm{~m} / \mathrm{s}$. A título de comparação, também se consideraram nos gráficos as curvas de queda livre no vácuo, geradas pelas equações horárias do Movimento Uniformemente Variado.

Na Figura 8, observa-se que, no intervalo de tempo considerado para a tomada de dados, a esfera de plástico não permite que se avalie o efeito do ar sobre seu movimento com a metodologia empregada, mesmo usando-se um smartphone que gravava a 120 fps. Assim, ela se mostrou inadequada para a proposta deste trabalho. O modelo teórico sugere que seria necessário algo em torno de dois segundos de queda para que se pudesse perceber o efeito do arrasto, mas isto significa que a altura inicial estaria próxima de $20 \mathrm{~m}$, fugindo do propósito do trabalho. Entretanto, pode-se observar que a esfera de isopor se adequou bem ao proposto. Nos gráficos das Figuras 9 e 10, o desvio relativo médio é 1,7\% para a posição e $2,1 \%$ para a velocidade. Em alguns lançamentos da bola de isopor, observou-se que possuía velocidade inicial negativa. De início, pensou-se em erro na tomada de dados, mas depois se lembrou de que o isopor eletriza com facilidade e poderia estar sendo atraído pela mão do lançador. Ocorre também que a superfície desta bola não é lisa, e a rugosidade, como se sabe, reduz o coeficiente de arrasto porque antecipa o regime turbulento. O ajuste das curvas em diversos ensaios com a bola de isopor ficou melhor quando se adotou $\mathrm{C}=0,45$.

Vale ressaltar-se que, no início do movimento, o módulo da velocidade e o número de Reynolds são pequenos, implicando que o arrasto poderia ser essencialmente viscoso, $F_{\text {arr }}=k . v$, mas transforma-se rapidamente em inercial, $F_{a r r}=k \cdot v^{2}$, devido ao aumento 

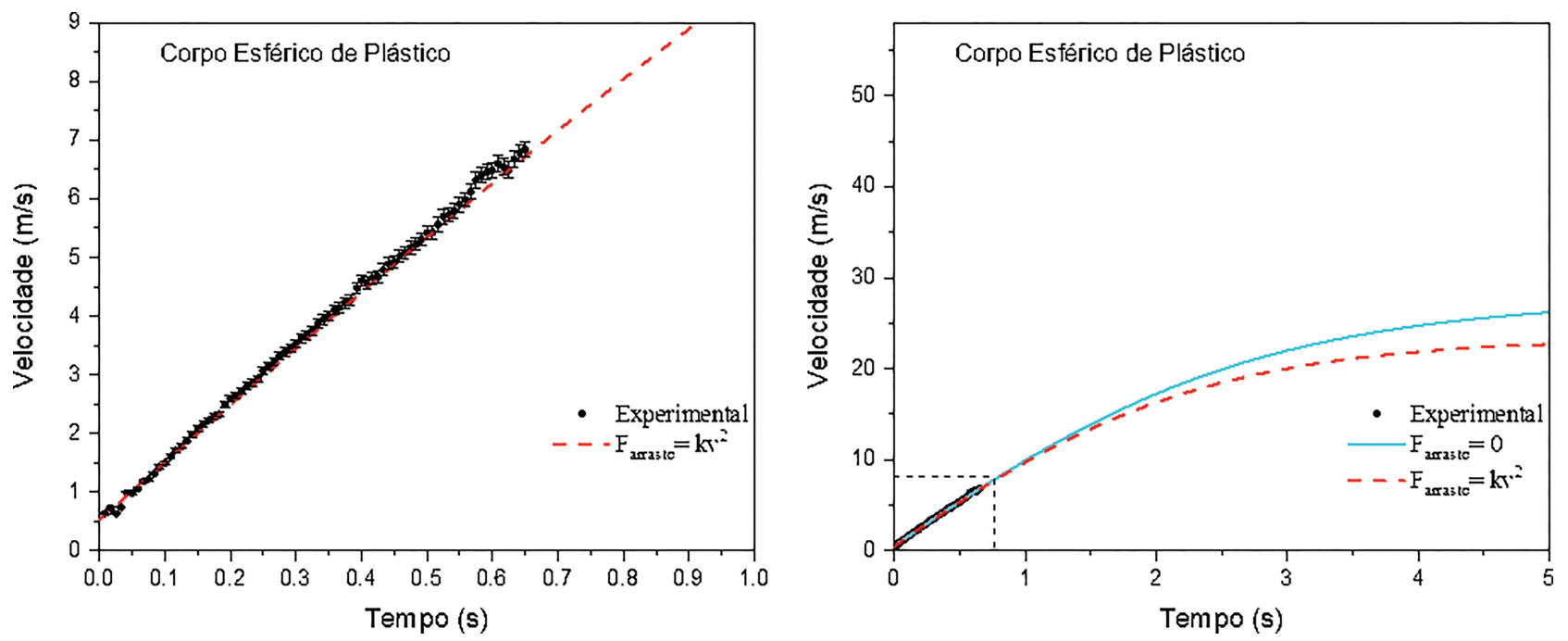

Figura 8: Gráficos comparativos da velocidade em função do tempo para a o corpo esférico de plástico. À direita, extrapolação das curvas teóricas.

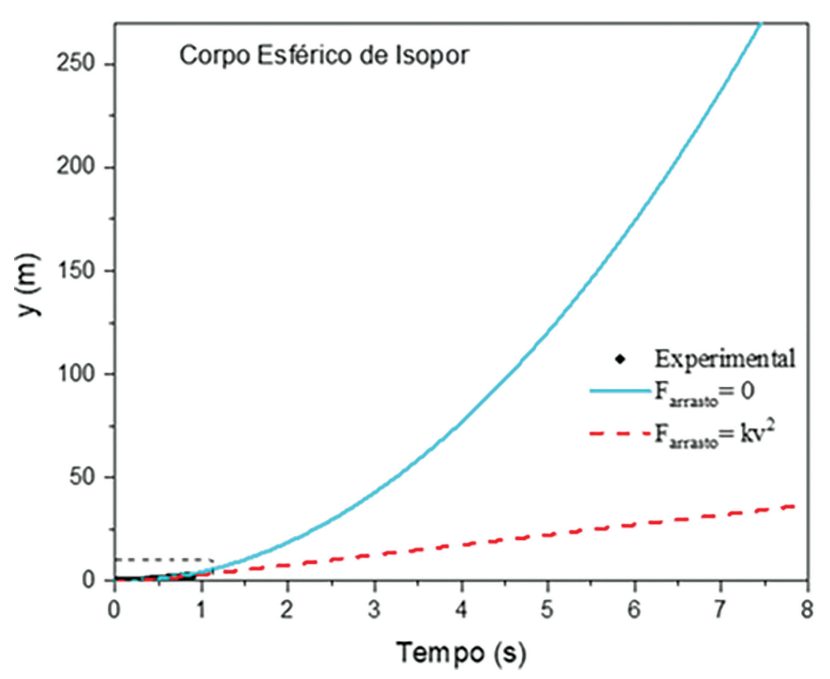

Figura 9: Gráficos comparativos da posição em função do tempo para a o corpo esférico de isopor. À direita, extrapolação das curvas teóricas.

da velocidade. Disso poderia inferir-se a necessidade de tratamentos distintos ao longo da trajetória, mas os resultados apresentados indicam que não é preciso. A justificativa teórica deste fato é que não há correlação entre o módulo da velocidade inicial com a magnitude da velocidade terminal, como prevê a equação (16), implicando que uma possível transição ocorrida do arrasto viscoso para o inercial não afetaria significativamente o comportamento da velocidade nos instantes seguintes. No intervalo $0,00 \mathrm{~s}$ a $0,30 \mathrm{~s}$, para o corpo de isopor $(\tau=0,49 s)$, e de $0,00 \mathrm{~s}$ a $0,50 \mathrm{~s}$ para o corpo de plástico $(\tau=2,82 s)$, é praticamente imperceptível a diferença entre as curvas com arrasto e sem arrasto. Ao final destes intervalos, as velocidades atingem valores da ordem de $3,0 \mathrm{~m} / \mathrm{s}$ e $5,0 \mathrm{~m} / \mathrm{s}$, respectivamente, e o número de
Reynolds seria $4.10^{3}$ para a o corpo de isopor e $1.10^{4}$ para o outro. Tais valores possibilitam concluir que o arrasto tornou-se inercial para ambas, e seu efeito é notório para o composto de isopor, cuja velocidade tende ao valor $v_{t}=4,6 \mathrm{~m} / \mathrm{s}$, observável nos gráficos da Figura 10

Uma prática comumente utilizada para a determinação do valor da aceleração gravitacional é a experiência de queda livre [39, 40]. Algumas destas práticas empregam uma esfera de aço, conectada inicialmente a um eletroímã, que sofre o efeito da histerese [41, 42. Via de regra, nestas práticas desconsidera-se o fenômeno de histerese e da resistência do ar para o cálculo de " $g$ ". Acaso seja desprezado o efeito do ar, e a aceleração seja calculada pela relação de Torricelli, $g_{\text {exper }}=v^{2} /(2 y)$, o valor obtido a partir dos dados experimentais será menor que o valor obtido para o vácuo, visto que o valor da velocidade, dado pela equação 22 , será menor devido à resistência do ar. Assim, a diferença relativa $\left(g_{T}-g_{\text {exper }}\right) / g_{T}$ entre o valor teórico, $g_{T} \cong 9,8 \mathrm{~m} / \mathrm{s}^{2}$, e o calculado será:

$$
\frac{\Delta g}{g_{T}}=1-\frac{v^{2}}{2 . g_{T} \cdot y}
$$

A Figura 11 apresenta a previsão do desvio relativo no cálculo do valor da aceleração calculado a partir da queda livre de uma pequena esfera de aço $\left(7,30 \mathrm{~g} / \mathrm{cm}^{3}\right)$ com 2,00 cm de diâmetro, em queda vertical no ar a partir do repouso. Considerou-se que o fluido era o ar, com os mesmos parâmetros das simulações anteriores. As equações 21 e (22) foram utilizadas para o cálculo do desvio definido pela equação (27). Do gráfico, é possível concluir-se que o efeito da resistência do ar para esta esfera será inferior a $0,1 \%$ para quedas de alturas inferiores a 2,0 m. Este resultado é coerente com o estudo que se fez com o corpo esférico de plástico, corroborando com estudos anteriores [43. 

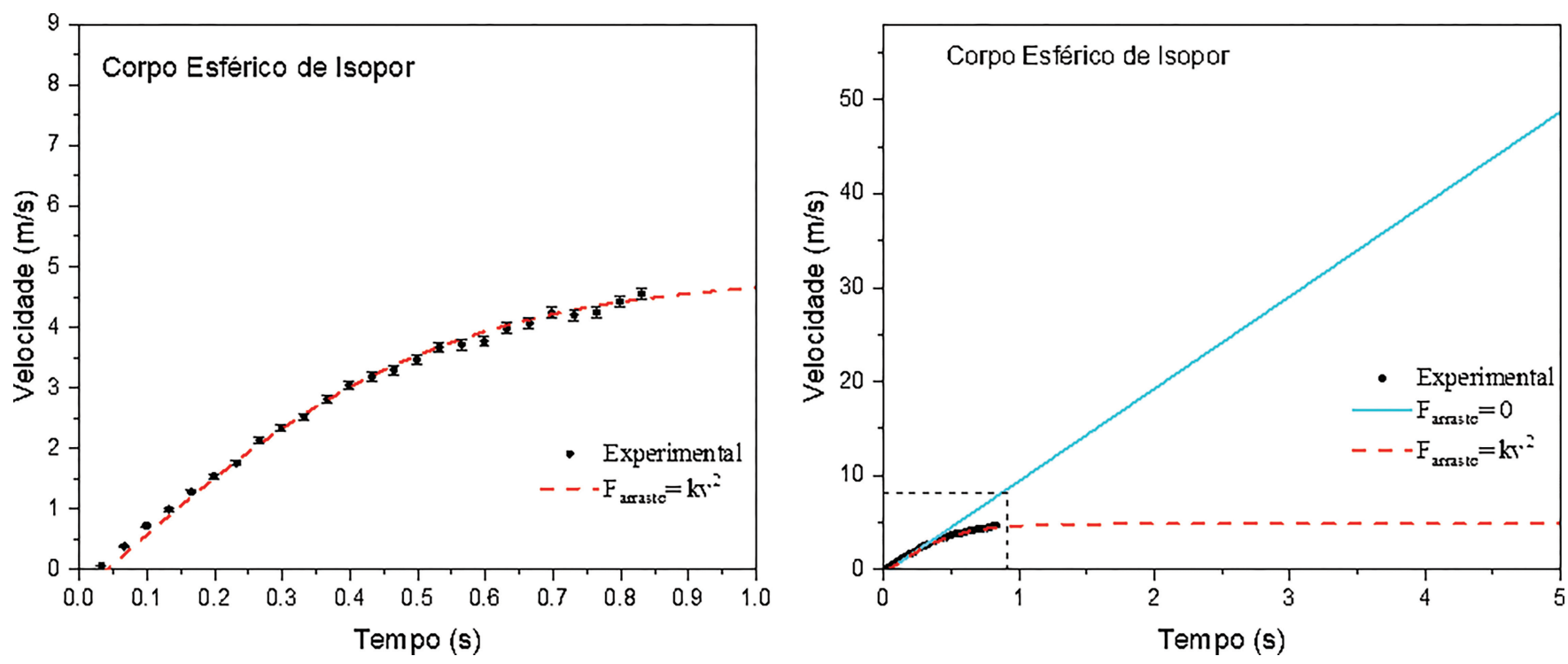

Figura 10: Gráficos comparativos da velocidade em função do tempo para a o corpo esférico de isopor. À direita, extrapolação das curvas teóricas.
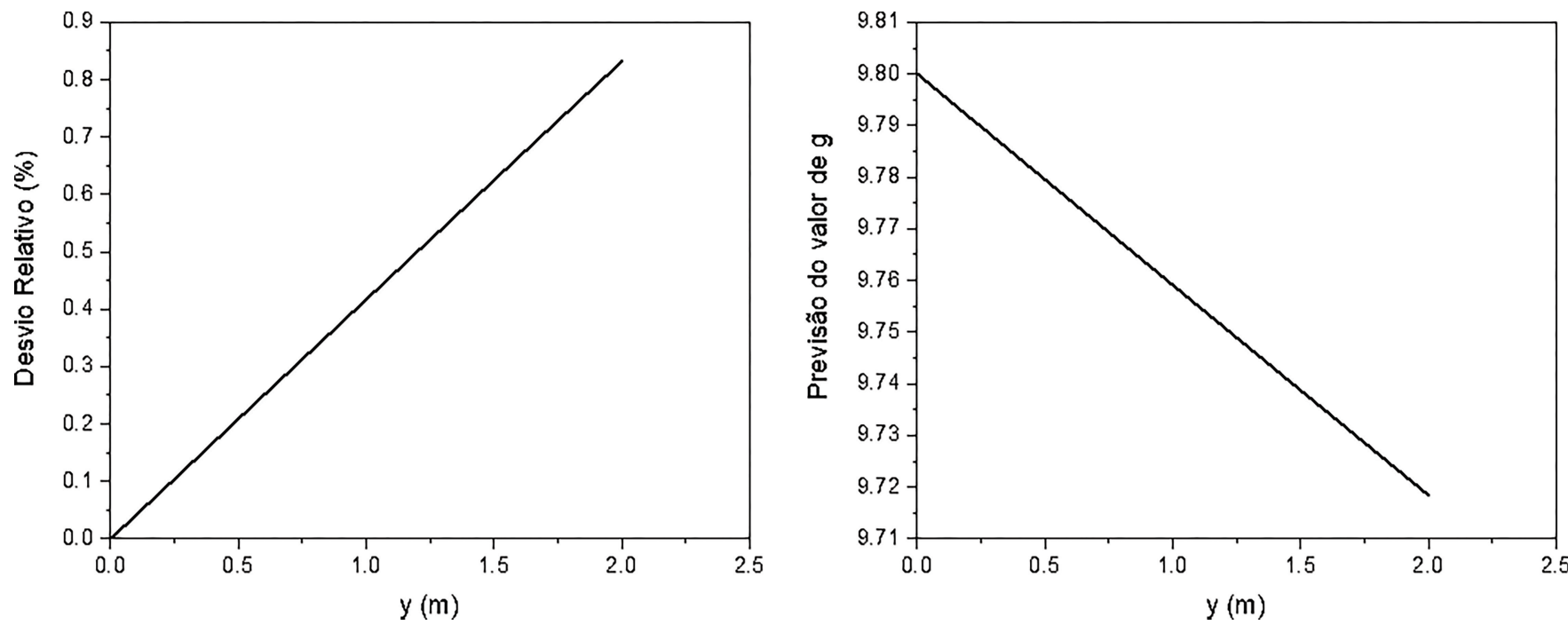

Figura 11: À esquerda, previsão do desvio relativo no cálculo da aceleração gravitacional para uma experiência de queda livre em função da altura devido ao efeito do ar. À direita, o valor que seria obtido acaso se considerasse o emprego da equação de Torricelli.

\section{Considerações finais}

Este trabalho mostrou que é possível apresentar-se aos estudantes as principais forças que regem a queda vertical de uma esfera de isopor considerando-se a presença do ar, e a partir destas deduzir-se as equações cinemáticas que descrevem o movimento. A modelagem teórica considerou uma situação bem realista, avançando no que comumente apresenta-se nos livros didáticos, nos quais a resistência do ar é desprezada ou pusilanimemente citada. Os gráficos das Figuras 9 e 10 indica que o modelo teórico empregado foi adequado para descrever a influência do ar no movimento de queda do corpo esférico de isopor. Por sua vez, o teste feito com o corpo esfera de plástico indicou que ela é inadequada para a atividade que se sugere, uma vez que não permitiu observar-se o efeito do arrasto e a velocidade terminal, considerando a altura de lançamento proposta no trabalho. Contudo, o corpo esférico de isopor mostrou-se muito bem adequado, validando a proposição. Na perspectiva didática, acredita-se ter contribuído para o processo de ensino e aprendizagem, apresentando-se um ensaio interessante e factível, envolvendo a inserção de tecnologias acessíveis, como softwares livres, microcomputador e smartphone, além do empregar materiais de baixo custo. O trabalho também oferece um caminho para se iniciar o estudo do movimento em fluidos, apresentando uma atividade prática que pode ser inserida nos cursos de 
graduação em ciências exatas. Alunos com conhecimento básico de Cálculo Diferencial e Integral poderão apreciar as deduções das equações, encontrando aqui um belo exercício de matemática. Um dos possíveis desdobramentos desse estudo seria realizar-se uma análise do efeito do ar em movimento de projéteis, entre outros.

\section{Referências}

[1] K. Weltner, M. Ingelman-Sundberg, A.S. Esperidião e P. Miranda, Rev. Bras. Ensino Fís. 23, 429 (2001).

[2] F.L. Silveira, Velocidade das pedras de granizo. (2015) Disponível em: https://www.if.ufrgs.br/ lang/ Textos/Granizo.pdf, acessado em 05 de março de 2020.

[3] S.M. Couto, A.E.O. Santos, S.M.J. Vieira, D.J.P. Silva, Rev. Bras. Eng. Agríc. Ambient. 8, 274 (2004).

[4] B. Leroy, Revis. Bras. Fís. 7, 693 (1977).

[5] C.E. Aguiar, G. Rubini, Rev. Bras. Ensino Fís. 26, 297 (2004).

[6] F.L. Silveira, Cad. Bras. Ens. Fís. 30, 156 (2013).

[7] J.O. Chang, F.L.Q. Costa, I.C. Dutra, G.A.F. Nery, L.H. Nery, E.R.R. Rodrigues, A.M.G. Selin, F.T. Zambello, D.J.P. Silva e R.R Cuzinatto, Cad. Bras. Ens. Fís. 36, 529 (2019).

[8] F.L. Silveira, A física no salto recorde de Felix Baumgartner, Rev. Bras. Ensino Fís. 37, 1 (2015).

[9] M.J. Carré, T. Asai, T. Akatsuka e J. Haake, Sport. Eng. 5, 193 (2002).

[10] P. Blanco, Phys. Teach. 56, 276 (2018).

[11] R. Cross, C. Lindsey, Phys. Teach. 52, 169 (2014).

[12] H.M. Nussenzveig, Curso de Física Básica. (Editora Blucher, São Paulo, 2013), v. 1. $5^{\mathrm{a}}$ ed.

[13] D. Halliday, R. Resnick e J. Walker, Fundamentos de Física - Mecânica (Editora LTC, Rio de Janeiro, 2014), $10^{\mathrm{a}} \mathrm{ed}$.

[14] R.A Freedman e H.D. Young, Física I: Mecânica (Editora Pearson, São Paulo, 2016), 14 ${ }^{\mathrm{a}}$ ed.

[15] P.A. Tipler, Física para Cientistas e Engenheiros (Editora LTC, Rio de Janeiro, 2019). $6^{\mathrm{a}}$ ed.

[16] W.H.C. Freire, M.L. Medeiros, D. Leite e R.M. Silva, Rev. Bras. Ensino Fís. 38, 1 (2016).

[17] B.S.G. Almeida e R.C. Silva, Rev. Bras. Ensino Fís. 37, 3505 (2015).

[18] J.M. Davies, J. Appl. Phys. 20, 821 (1949).

[19] Open Source Physics, TRACKER - Video analysis and modeling tool., (2009). Disponível em: https:// physlets.org/tracker/download/AAPT_video_modeling 2009.pdf, acessado em 23 de março de 2020.

[20] R.W. Fox, A.T. McDonald, P.J. Pritchard e J.W. Michtell, Introdução à mecânica dos fluidos (Editora LTC, Rio de Janeiro, 1998), $4^{\mathrm{a}}$ ed.

[21] H.M. Nussenzveig, Curso de Física Básica, Fluidos, Oscilações e Calor (Editora Blucher, São Paulo, 2014), $5^{\mathrm{a}}$ ed.

[22] G.K. Batchelor, An introduction to fluid dynamics (Cambridge University Press, Cambridge, 2002).

[23] E. ToolBox, International Standard Atmosphere, (2005). Disponível em: https://www.engineeringtoolbox.com/in ternational-standard-atmosphere-d_985.html, acessado em 27 de março de 2020.
[24] H.P. Bohm, A general equation for the terminal fall speed of solid hydrometeors, J. Atmos. Sci. 46, 2419 (1989).

[25] T. Kroetz, Rev. Bras. Ensino Fís. 35, 3308 (2013).

[26] T.R. Barros e W.S. Dias, Rev. Bras. Ensino Fís. 41, e20190049 (2019).

[27] R.V. Giles, Mecânica dos fluidos e hidráulica (Editora McGraw - Hill, São Paulo, 1980), $2^{\text {a }}$ ed.

[28] V.L.B. Jesus e D.G.G. Sasaki, Rev. Bras. Ensino Fís. 36, 1 (2014).

[29] M.A.J. Barros e V.L.B. Jesus, Rev. Prof. Fís. 3, 48 (2019).

[30] D. Brown, Tracker - Video Analysis and Modeling Tool, (2020). Disponível em: https://physlets.org/tracker/ acessado em 15 de maio de 2020.

[31] Laboratório Didático de Fìsica - UFRGS, Analisando imagens e vídeos com o computador, (2009). Disponível em: http://www.if.ufrgs.br/cref/uab/lab/tracker.html acessado em 15 de maio de 2020 .

[32] D. Brown, Getting Started with Tracker, (2017). Disponível em: https://www.youtube.com/watch?v=La 3H7JywgX0, acessado em 15 de maio de 2020.

[33] N.S. Wendt, Utilizando o Software Tracker para o Ensino da Física, (2019). Disponível em: https://www.youtube. $\mathrm{com} /$ watch $\mathrm{v}=2 \mathrm{M} 3 \mathrm{IPON} 5 \mathrm{pZM}$, acessado em 15 de maio de 2020.

[34] J. Stephens, M. Bostjancic e T. Koskulitz, Phys. Teach. 57, 193 (2019).

[35] T. Martin, K. Frisch e J. Zwart, Phys. Teach. 58, 195 (2020).

[36] V.L.B. Jesus, Experimentos e videoanálise - dinâmica (Editora Livraria da Física, São Paulo, 2014), $1^{a}$ ed.

[37] R. Boynton, Precise Measurement of Mass, 60th Annu. Conf. Arlington, Texas, May 19-23. (2001) Disponível em: https://www.sawe.org/papers/3147/buy

[38] INSTITUTO NACIONAL DE METEOROLOGIA, Dados Cartográficos, (2020). Disponível em: http:// www.inmet.gov.br/portal/index.php?r=estacoes/estaco esConvencionais, acessado em 4 de luho de 2020.

[39] L.A. Ribeiro Junior, M.F. Cunha e C.C. Rev. Bras. Ensino Fís. 34, 4602 (2012).

[40] L.O.Q. Peduzzi, A.B. Clebsch, Uma experiência sobre galileu em um curso de licenciatura em fisica na modalidade à distância, (2007). Disponível em: http://www.nutes.ufrj.br/abrapec/vienpec/CR2/p900. pdf, acessado em 27 de março de 2020.

[41] V.S. Leite e W. Figueiredo, Rev. Bras. Ensino Fís. 29, 53 (2007).

[42] https://azeheb.com.br/acessorios-para-queda-livre-bas ic.html, acessado em 27 de março de 2020.

[43] P. Mohazzabi, Phys. Teach. 56, 168 (2018). 